\title{
Bibliotecas universitarias 2.0. El caso de España
}

\author{
José Luis Herrera Morillas \\ Ana Castillo Díaz *
}

Artículo recibido:

7 de abril de 2011.

Artículo aceptado:

12 de enero de 2012.

\section{RESUMEN}

En este artículo se recopilan los recursos de la Web 2.0 utilizados por las bibliotecas universitarias españolas y se analizan su finalidad y principales rasgos. Se combina la metodología de la visualización en línea y el análisis de contenido para estudiar la utilización que de dichos recursos hacen las 63 bibliotecas universitarias españolas. Entre las herramientas más empleadas destacan el acceso al OPAC 2.0, las redes sociales y los blogs. Por otra parte, entre los recursos menos empleados se encuentran los marcadores sociales y las plataformas para compartir archivos de imágenes o videos, como Flickr o YouTube, entre otros.

* Ambas autores pertenecen a la Universidad de Extremadura, España. (José L.: jlhermor@unex.es); (Ana: acasdia@alcazaba.unex.es)

INVESTIGACIÓN BiBLIOTECOLÓGICA, Vol. 25, Núm. 55, septiembre/diciembre, 2011, México, ISSN: 0187-358X. pp. 175-200 
Palabras clave: Bibliotecas universitarias; Web 2.0; Bibliotecas 2.0; Bibliotecas españolas.

\begin{abstract}
University libraries 2.0. The case of Spain

José Luis Herrera Morillas and Ana Castillo Díaz

This paper aims to explore the status and construction pattern of Web 2.0 technologies employed in Spanish university libraries, its purpose and features. A combination of online survey and content analysis methodology is applied to 63 Spanish university libraries' websites. The most used 2.0 tools are OPAC, social networks and blogs, while the less abundant are the folksonomies and webs to share files such as YouTube or Flickr.
\end{abstract}

Keywords: University libraries; Web 2.0; Libraries 2.0; Spanish libraries.

\title{
I. INTRODUCCIÓN
}

\subsection{Objetivos y metodología}

$\mathrm{E}$ n no pocas ocasiones las organizaciones e instituciones, entre ellas las bibliotecas, han comenzado a incorporar herramientas catalogadas como 2.0 en sus webs corporativas. Así, es frecuente encontrar acceso a blogs, wikis, redes sociales, etc. desde las páginas web de las bibliotecas de todo el mundo pero, ¿qué uso se está llevando a cabo de esas herramientas? ¿Están exprimiendo correctamente su potencial? Es evidente que aunque una buena gestión de ellas puede proporcionar una imagen positiva, el simple hecho de incluirlas como parte de la comunicación no garantiza buenos resultados. Es cierto que en ocasiones es mejor no estar en esos lugares que presentar espacios descuidados y desactualizados que pueden afectar la reputación de la biblioteca y, por ende, a la de la institución que hay tras ella.

Este trabajo muestra un análisis de la utilización que hacen de estos recursos las bibliotecas universitarias españolas. Siguiendo estudios precedentes (Han, Quan Liu, 2009; Linch, 2008) se ha realizado una revisión no sólo 
de la utilización de las herramientas 2.0 que se emplean, sino también de las características que éstas tienen y de la finalidad para las que son empleadas.

Esta investigación traza un cuadro general sobre el uso de los recursos de la web 2.0 en las bibliotecas universitarias españolas en un momento dado, y puede servir de ayuda a los usuarios para conocer qué bibliotecas y qué recursos de estas características se ofrecen en España. También, puede serle útil a las bibliotecas que quieran iniciar o mejorar el empleo de recursos de la web 2.0.

\subsection{La importancia de la comunicación corporativa en las universidades}

Existen tres planteamientos clave que deberían estar presentes en todo proyecto universitario que pretenda alcanzar el éxito en su sector:

- Prestar un servicio de calidad.

- Ofrecer servicios adaptados a las exigencias de sus públicos.

- Establecer una comunicación puntual y apropiada con sus públicos internos y externos.

Esta tríada supondría el paso previo, y común a todas las universidades, a cualquier planteamiento de diferenciación.

Una diferenciación positiva no es posible sin un servicio de calidad que satisfaga las demandas de los públicos, pero tampoco puede darse sin el establecimiento de una comunicación que contribuya a conjugar la realidad que la institución es y desea transmitir, con la realidad que los públicos divisan e interpretan.

Las bibliotecas universitarias, como servicio muy importante que son dentro de las instituciones educativas universitarias, deberían cuidar estos aspectos.

En el interior de la organización la comunicación es útil para que se asuman y se pongan objetivos en común, que favorezcan también el trabajo de sus distintos miembros en un mismo sentido. En el ámbito externo tal comunicación constituye una pieza clave para dar a conocer los servicios y facilitar los intercambios con los públicos.

Además, a través de la comunicación, la organización puede detectar las demandas de sus interlocutores y enriquecer de ese modo sus futuros planteamientos empresariales.

En resumen, la comunicación institucional se constituye como una herramienta provechosa para la Universidad, y por extensión para sus servicios bibliotecarios, en lo que se refiere a (Castillo, 2007): 
- La transmisión de su realidad.

- La aprobación por parte de la sociedad (legitimidad social).

- La detección de las nuevas demandas.

- La motivación y cohesión de los recursos humanos.

La Universidad no constituye ninguna excepción con respecto a otras instituciones y para consolidar su legitimidad social debe transformarse, evitando que en su evolución la Sociedad del Conocimiento la relegue a una posición marginal. Cuena (en Marín et al., 2005) explica que:

la Universidad actual recoge en su declaración de misión y funciones: la creación, el desarrollo, la transmisión y la crítica de la ciencia, de la técnica, de la cultura y del arte, orientadas hacia la libertad, el desarrollo sostenible, la justicia, la paz y la amistad entre los pueblos. En su filosofía está el apoyo científico y técnico al desarrollo cultural, social y económico en todos sus ámbitos. Esta nueva proyección exige a la Universidad un esfuerzo de adaptación a las transformaciones de su entorno y a las suyas propias.

A pesar de su peso la aprobación social no es suficiente para triunfar en el mercado universitario actual. Para atraer a sus públicos de interés y para entablar y mantener una relación fructuosa y duradera con ellos, es imprescindible que la Universidad destaque en algún aspecto que interese a sus stakebolders (quienes apuestan por ella). Éstos no dudan en desplazarse, nacional e internacionalmente, en busca de las ofertas que mejor se adapten a las particulares y ambiciosas demandas del mercado (Cant, 2004).

En términos generales desde la aparición de esos primeros gabinetes de prensa universitarios, la realidad comunicativa de este sector ha variado sensiblemente y caminado hacia una mayor adecuación de los contenidos a los intereses particulares de cada grupo de usuarios. Internet y la World Wide Web han facilitado y multiplicado las posibilidades de los discursos de las marcas universitarias.

Como cualquier otra institución, las universidades deben emplear las distintas herramientas de comunicación a su alcance desde una visión unificada, atendiendo a la política general de la institución y tomando como punto de partida la identidad establecida.

Además, la comunicación en-línea universitaria debe incardinarse dentro del plan de comunicación general de la institución, el cual habrá de diseñarse de acuerdo con los criterios establecidos por la estrategia general de negocios. Asimismo, en este marco competitivo universal, conviene analizar y evaluar las oportunidades existentes y las amenazas que suponen las actividades de 
comunicación desarrolladas en el entorno regional, nacional e incluso europeo e internacional por otras instituciones y empresas rivales.

\subsection{Comunicación en-línea de las bibliotecas universitarias}

Las bibliotecas constituyen una parte fundamental de las universidades y tienen una misión muy importante puesto que se trata de uno de los servicios más demandados por profesores y alumnos. Por esta razón resulta especialmente relevante cuidar la comunicación de este servicio.

Las bibliotecas están empleando las herramientas sociales para comunicarse con sus usuarios. En el caso concreto de España las bibliotecas universitarias suelen emplear en gran medida las redes sociales, en concreto Facebook (Margaix-Arnal, 2008):

Por este motivo las bibliotecas han comenzado a plantearse su presencia en estos sitios: para estar donde los usuarios están, utilizar las mismas plataformas y canales de comunicación que ellos y seguir siendo relevantes en el contexto de su experiencia del uso de Internet.

\subsection{Web 2.0, bibliotecas 2.0: estado de la cuestión}

Desde hace ya algún tiempo se viene calificando como 2.0 al uso de las herramientas que facilitan la comunicación entre las distintas organizaciones y sus públicos. Así, es frecuente encontrar denominaciones como marketing 2.0, periodismo 2.0, o cultura 2.0 entre otros. El origen del término se le atribuye a Tim O'Reilly, (O'Reilly, 2005).

Coombs (2007) define el concepto como

a space that allows anyone to create and share information online - a space for collaboration, conversation, and interaction; a space that is highly dynamic, flexible, and adaptable. ${ }^{1}$

Esta idea más simplificada de crear y compartir información en el entorno en-línea es la que subyace siempre tras el apellido 2.0.

Muchas instituciones están empleando este tipo de herramientas para comunicarse con sus audiencias, especialmente si se trata de jóvenes pertenecientes a la Generación Einstein, (Boschma, 2006).

1 “...un espacio que le permite a cualquiera crear y compartir información en-línea; un espacio para la colaboración, la conversación y la interacción; un espacio que es altamente dinámico, flexible y adaptable". Trad. del C. 


\begin{tabular}{|c|c|c|}
\hline 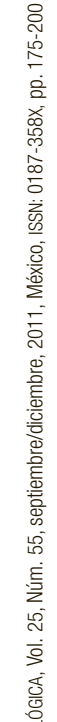 & \multicolumn{2}{|c|}{$\begin{array}{l}\text { Las bibliotecas no son una excepción en este ámbito (Breeding, 2007). } \\
\text { Son numerosos los bibliotecarios que actualizan casi diariamente sus blogs } \\
\text { (anuarios de la Fundación Alonso Quijano) y mantienen una comunicación } \\
\text { directa con sus usuarios. Incluso una comunicación multidireccional, que } \\
\text { supone el contacto entre usuarios que no se conocían previamente. } \\
\text { Considerando la utilización de este tipo de herramientas algunos autores } \\
\text { (Chad, 2005; Maness, 2006) aplican el concepto } 2.0 \text { a las bibliotecas, defi- } \\
\text { niéndolas como bibliotecas } 2.0 \text {. } \\
\text { El término fue empleado por primera vez por Michael Casey en su blog } \\
\text { (Maness, 2006). Según Maness la biblioteca } 2.0 \text { consiste en "la aplicación de } \\
\text { tecnologías web interactivas, colaborativas y multimedia a colecciones y ser- } \\
\text { vicios bibliotecarios basados en la web". Este tipo de aplicaciones les permite } \\
\text { a las bibliotecas promover y extender sus servicios. } \\
\text { Gran parte de la literatura sobre la materia coincide en señalar una serie } \\
\text { de herramientas como propias de la web 2.0. Y son las que analizan la mayo- } \\
\text { ría de estudios sobre las bibliotecas } 2.0 \text {. }\end{array}$} \\
\hline$\overline{\overline{\mathbf{m}}}$ & Herramientas & Definición \\
\hline & RSS (sindicación realmente simple) & $\begin{array}{l}\text { Es una forma muy sencilla para recibir, directamente en un ordenador } \\
\text { o en una página web (a través de un lector RSS) información actua- } \\
\text { lizada sobre páginas web favoritas de los usuarios, sin necesidad de } \\
\text { que tengan que visitarlas una a una. }\end{array}$ \\
\hline & Blogs y microblogs & $\begin{array}{l}\text { Los microblogs o nanoblogs permiten a los usuarios enviar mensajes } \\
\text { de texto con una longitud máxima de } 140 \text { caracteres de forma instan- } \\
\text { tánea y gratuita. Twitter se ha convertido en el líder indiscutible del } \\
\text { microblogging, (Celaya, 2008). }\end{array}$ \\
\hline & Wiki & $\begin{array}{l}\text { Es un sitio web colaborativo que se puede editar de manera conjunta } \\
\text { por varios usuarios. En el ámbito empresarial se utilizan para inter- } \\
\text { cambiar ideas, como agenda de reuniones, para atender al cliente } 0 \\
\text { darle soporte a un producto, (Celaya y Herrera, 2007). }\end{array}$ \\
\hline & Podcast & $\begin{array}{l}\text { El podcasting consiste en la distribución de archivos multimedia } \\
\text { (usualmente de audio) mediante un sistema que permita suscribirse } \\
\text { (RSS) y usar un programa que lo descarga para que el usuario lo es- } \\
\text { cuche en el momento que quiera, (Georghegan y Klass, 2005). }\end{array}$ \\
\hline & Contenidos de video (Youtube) & Sitio web en el que los usuarios pueden compartir y visualizar videos. \\
\hline & $\begin{array}{l}\text { Contenidos de imágenes (Flickr, } \\
\text { Picassa) }\end{array}$ & Sitios webs en los que los usuarios pueden compartir fotografías. \\
\hline & Mensajería instantánea & $\begin{array}{l}\text { Se trata de una forma de comunicación en tiempo real, entre dos } 0 \\
\text { más personas basada en texto. }\end{array}$ \\
\hline & Marcadores sociales & $\begin{array}{l}\text { Se trata de un servicio, por lo general gratuito, para almacenar, eti- } \\
\text { quetar y compartir la información. Cualquier persona que esté inte- } \\
\text { resada en un determinado tema puede encontrar mucha información } \\
\text { sobre el mismo de forma rápida y sencilla. Ejemplo: Delicious. }\end{array}$ \\
\hline
\end{tabular}


Margaix-Arnal (2007) recoge una revisión de la utilización del término 2.0 y propone una serie de elementos básicos de la biblioteca 2.0, que agrupa en tres niveles:

1. Herramientas
a. Blogs
b. Wikis
c. Rss ${ }^{2}$
d. Software social
e. Mashups ${ }^{3}$

2. Contenido social
a. Comentarios
b. Etiquetas
c. Ratings ${ }^{4}$
d. Otras aportaciones

3. Actitudes
a. Aprovechamiento de la inteligencia colectiva
b. Confianza radical
c. Mejora continua

De acuerdo con este autor las herramientas son sólo una parte de la web 2.0; sin generar contenidos de interés y una actitud activa no se aprovecharía el enorme potencial de utilidades que son bastante sencillas para el usuario.

Algunos autores se han centrado en el análisis de la aplicación de determinadas herramientas para las bibliotecas: mensajería instantánea (Foley, 2002), wikis (Boeninger, 2006; Fichter, 2006), RSS (Harinarayana, 2007), podcasts (Jowitt, 2007; Abram, 2006). Habib (2006) llega a proponer un marco metodológico para orientar el empleo de las herramientas 2.0 en las bibliotecas académicas.

En su investigación Chua y Goh (2010) tratan de dar respuesta a las siguientes cuestiones: a) ¿En qué medida prevalecen las aplicaciones 2.0 en las bibliotecas?; b) ¿En qué sentido son usadas las aplicaciones 2.0 en las bibliotecas?;

2 RSS Really simple syndication. Sindicación (o asociación) realmente simple. Trad. del C.

3 Una mashup es una página web o aplicación que usa y combina datos, presentaciones y funcionalidad procedentes de una o más fuentes para crear nuevos servicios.

4 Ratings. Clasificaciones por rangos o clases. Trad. del C. 
y c) ¿La presencia de las aplicaciones 2.0 fomenta la calidad de las webs de bibliotecas? Los autores analizaron 120 bibliotecas de Norteamérica, Europa y Asia. Los resultados muestran que el orden de popularidad de uso de los recursos 2.0 implementados por las bibliotecas son: blogs, RSS, mensajería instantánea, servicios de redes sociales y aplicaciones de etiquetado.

Harinarayana et al. (2010), muestran un análisis de la utilización de las herramientas 2.0 por parte de las bibliotecas sobre las 100 principales universidades del mundo (Times Higher Education). Su investigación trata de conocer los tipos de tecnologías 2.0 que son aplicadas en las webs de las bibliotecas universitarias y para qué propósitos son usadas. Los contenidos analizados fueron los siguientes:

1. RSS (sindicación o asociación realmente simple).

2. Blogs, bitácoras o diarios online.

3. Wikis.

4. Contenidos de audio y vídeo (podcasts /vídeo streaming).

5. Mensajería instantánea.

6. Marcadores sociales (entendidos como un método empleado por los usuarios para almacenar, organizar, buscar y gestionar páginas de Internet a través del uso de etiquetas.

7. Redes sociales en-línea.

Los resultados del estudio muestran que el 37\% de las bibliotecas universitarias usan fuentes RSS para la difusión de sus eventos, también el 37\% de tales instituciones instituciones emplean servicios de mensajería instantánea en sus web corporativas. Por su parte el $15 \%$ provee espacio de blogs para sus usuarios. Entre los servicios menos populares están el podcasting (el 3\% de las universidades) y el de vidcasting (el 6\% de ellas).

Realizando una revisión de los recursos que comparten ambos estudios se puede concluir que las herramientas más empleadas por parte de las bibliotecas universitarias son las fuentes RSS y la mensajería instantánea. El primer servicio agiliza la actualización de la información sobre cuestiones de interés para los usuarios y el segundo aporta fluidez a la comunicación entre los bibliotecarios y los usuarios.

\begin{tabular}{|l|l|}
\hline \multicolumn{2}{|c|}{ Herramientas 2.0 usadas en las bibliotecas universitarias } \\
\hline Chua y Goh (2010) & Harinarayana (2010) \\
\hline Blogs & RSS \\
\hline Rss & Mensajería instantánea \\
\hline Mensajería instantánea & Blogs \\
\hline
\end{tabular}




\begin{tabular}{|l|l|}
\hline Redes sociales & Podcasting \\
\hline Etiquetado & Vidcasting \\
\hline
\end{tabular}

Fuente: elaboración propia a partir de Chua y Goh (2010) y Harinarayana (2010).

\section{Metodología}

En la metodología utilizada se ha tenido en cuenta la investigación de Han y Quan Liu (2009) para las bibliotecas universitarias de China, en la que se combina la estrategia de visualización en-línea y el análisis de contenido de los sitios web de las bibliotecas, estudiando un listado de datos específicos de una variedad de categorías. Este trabajo a su vez está basado en un estudio anterior de Linch (2008) dedicado a las bibliotecas universitarias de Australia. En ambos se parte de una categorización de los recursos 2.0 a los que se les aplica una lista de evaluación. Los datos de esta lista distinguen para cada categoría entre finalidad y rasgos. Se han tomado como base estas investigaciones por la proximidad al objeto de nuestro estudio: las bibliotecas universitarias de un país. En ambos casos se realiza un análisis de los recursos que se pretendían revisar en el caso español.

La categorización y lista de evaluación se basan en las que fueron descritas por estos autores adaptándolas a la realidad de las bibliotecas universitarias españolas. Esta adaptación ha consistido, fundamentalmente, en eliminar los elementos poco significativos (por no estar presentes en las bibliotecas analizadas) y en incluir algunos elementos para reflejar cualidades que sí son frecuentes en las bibliotecas españolas. Para ello se llevó a cabo una visualización previa del conjunto de los sitios web antes de definir las categorías y los elementos de la lista de evaluación.

Las 7 categorías establecidas para el análisis se corresponden con los siguientes recursos:

- Opac $2.0^{5}$

- RSS

- Blog

- Mensajería instantánea

- Redes sociales

- Compartir archivos multimedia

- Otros

5 Esta variable incluye, en el trabajo de Han y Quan Liu (2009), las herramientas de navegación web, la nube de etiquetas y las alertas textuales. 
La lista de evaluación consta de un total de 64 elementos que se reproducen en la Tabla I y representan el 100\% de las bibliotecas universitarias españolas que emplean recursos propios de la web 2.0.

En la categoría redes sociales los elementos de evaluación se aplican a la red Facebook, por ser la red de carácter generalista más empleada por las universidades españolas (Margaix-Arnal, 2008a). Además, se indica si las bibliotecas participan en otras redes de uso más reducido.

La categoría "otros" es una variable abierta que contempla otro tipo de recursos de la web 2.0 que están presentes pero de manera menos significativa.

El listado de las bibliotecas universitarias españolas se ha obtenido del directorio Bibliotecas universitarias y de investigación españolas: $<\mathrm{http} / / /$ bibliotecas.csic.es/otroscat/webuni.html> elaborado por el CSIC (Consejo Superior de Investigaciones Científicas).

Las consultas de los sitios webs se han llevado a cabo durante dos momentos: el primero en julio de 2011 y el segundo en la primera quincena de octubre del 2011.

El modelo más difundido de presencia en la web de estas bibliotecas se da a través de un sitio web que concentra la información y los recursos. Estos sitios son los que ocupan la presente investigación. Sin embargo existen excepciones a este modelo: hay bibliotecas que además de la web general de la biblioteca universitaria cuentan con sitios webs diferenciados para cada biblioteca específica, y en algunos casos éstas disponen de recursos propios. Esta situación se ha tenido en cuenta, de tal modo que se han incluido en el análisis los respectivos sitios web, cuando no existían recursos 2.0 en el sitio web de la biblioteca general y sí en ellos. Estos casos se han indicado oportunamente mediante una nota a pie de página en la Tabla 1, donde se recoge la lista de evaluación con los datos extraídos.

Los indicadores analizados en cada uno de los parámetros observados son los que se detallan a continuación:

- Finalidad y rasgos del OPAC 2.0 en las bibliotecas universitarias españolas

El OPAC 2.0 se puede entender como "la aplicación de las tecnologías y las actitudes de la Web 2.0 al catálogo bibliográfico", (Margaix-Arnal, 2008b).

Casey y Savastinuk $(2006)^{6}$ justifican su utilidad y delimitan las aportaciones que la Web 2.0 puede hacer a los OPACs afirmando que los 
comentarios, las etiquetas y las puntuaciones de los usuarios permiten crear un producto con más información para los siguientes usuarios. Aspectos analizados:

\begin{tabular}{|l|}
\hline \multicolumn{1}{|c|}{ Finalidad } \\
\hline $\begin{array}{l}\text {-Añadir información al Opac tradicional } \\
\text {-Cambio al Opac 2.0 }\end{array}$ \\
\hline \multicolumn{1}{|c|}{ Rasgos } \\
\hline $\begin{array}{l}\text {-Herramientas para buscar en la Red } \\
\text {-Usuarios que crean sus propias listas de libros } \\
\text {-Incluir etiquetas, puntuaciones y comentarios } \\
\text {-Registros que añaden a la cubierta del libro, sumario, etc. } \\
\text {-Ordenación de resultados según su información social } \\
\text {-Enlaces a recursos de información }\end{array}$ \\
\hline
\end{tabular}

- Finalidad y rasgos de RSS en las bibliotecas universitarias españolas El RSS (Rich site summary o really simple syndication) tiene por finalidad la distribución masiva de información procedente de distintos sitios. Un archivo RSS se suele definir como una descripción estructurada de uno o varios sitios web. Para poder interpretar estos archivos hay que utilizar programas agregadores. Se pueden utilizar diferentes posibilidades para la lectura de estos archivos, algunas incorporadas en los navegadores, otras, están en-línea y otras se tienen que instalar (Dobrecky, 2007).

De cara al público en general la utilidad de RSS radica en poder suscribirse a muchos contenidos de páginas web "sin la necesidad de visitar cada uno de los lugares que está interesado y chequear por actualizaciones" (Ríos, 2009).

Aspectos analizados:

\begin{tabular}{|l|}
\hline \multicolumn{1}{|c|}{ Finalidad } \\
\hline -Noticias y eventos de la biblioteca \\
-Novedades del catálogo \\
-Otros \\
\hline \multicolumn{1}{c|}{ Rasgos } \\
\hline $\begin{array}{l}\text {-Enlaces a páginas para descargar lectores RSS } \\
\text {-Noticias clasificadas por temas, fecha o título } \\
\text {-Instrucciones sobre uso } \\
\text {-Las noticias se pueden buscar }\end{array}$ \\
\hline
\end{tabular}

- Finalidad y rasgos del blog en las bibliotecas universitarias españolas El blog se ha constituido como una de las herramientas 2.0 más utilizada 
hasta el momento por las bibliotecas (González, 2007). Lo más característico son las anotaciones individuales de periodicidad variable, ordenadas generalmente de forma cronológica inversa y de las cuales se conserva un archivo (Leiva, 2006).

Para Andreu et al. (2007), la actualización frecuente que permite esta herramienta se puede traducir en un incremento de las visitas a la web de la biblioteca. Los usos más importantes que se le pueden dar los sintetiza el Bloc de Reserva de la Universitat de Barcelona $<$ http:// blocbibreserva.ub.edu/>:

El bloc de Reserva té la voluntat de convertir-se en un espai de comunicació i difusió del fons, les novetats, els recursos, els serveis i les activitats de la Biblioteca de Reserva de la Universitat de Barcelona així com de notícies sobre el món del llibre antic.

Aspectos analizados:

\begin{tabular}{|l|}
\hline \multicolumn{1}{|c|}{ Finalidad } \\
\hline -Apoyo a los servicios bibliotecarios \\
-Informa sobre novedades y noticias de la biblioteca \\
-Participación, comunicación de los usuarios \\
-Uso interno de los bibliotecarios \\
-Otros \\
\hline \multicolumn{1}{|c|}{ Rasgos } \\
\hline -Tiene más de un blog \\
-Incluye enlace a la página de la biblioteca \\
-Tiene archivos \\
-RSS de las entradas y comentarios \\
-Se pueden buscar las entradas por temas, categorías \\
-Se pueden buscar las entradas por fecha \\
-Las entradas se pueden recuperar por palabras clave \\
-Hay fecha en los post \\
-Las entradas archivadas superan un año \\
-Los últimos post están entre los dos últimos días \\
-Se incluyen enlaces a recursos relevantes \\
-Incluye enlaces a blogs similares
\end{tabular}

- Finalidad y rasgos de la mensajería instantánea en las bibliotecas universitarias españolas

Para Borrell y Maciel (2009) el chat se enmarca dentro del aprovechamiento que las bibliotecas están haciendo de los entornos virtuales para ofrecer información al usuario sin que tenga que desplazarse hasta la biblioteca, "esto puede hacerlo a través de las distintas opciones como mail, formularios electrónicos o chat, entre los más difundidos". Se trata de un sistema telemático que permite realizar a los usuarios sus consultas a través de Internet, y que aporta la gran ventaja de ofrecer 
conexiones en directo (Merlo, 2005) frente a otros procedimientos anteriores.

Aspectos analizados

\begin{tabular}{|l|}
\hline \multicolumn{1}{|c|}{ Finalidad } \\
\hline $\begin{array}{l}\text {-Apoyo al servicio de referencia } \\
\text {-Orienta sobre los servicios de la biblioteca }\end{array}$ \\
\hline \multicolumn{1}{c|}{ Rasgos } \\
\hline -Chat a base de texto \\
-Chat con voz \\
-Servicio disponible 8 horas al día \\
-Servicio disponible más de 8 horas al día
\end{tabular}

- Finalidad y rasgos de Facebook las redes sociales en las bibliotecas universitarias españolas

Las redes sociales son una de las manifestaciones más novedosas y difundidas del mundo 2.0. Se caracterizan por la interacción social entre individuos, grupos e instituciones. Son sistemas abiertos y en construcción permanente que "involucran a conjuntos que se identifican en las mismas necesidades y problemáticas y que se organizan para potenciar sus recursos" (Ríos, 2009).

Por ello no nos puede extrañar que las bibliotecas estén incrementando su incorporación a estas redes para "estar donde los usuarios están, utilizar las mismas plataformas y canales de comunicación que ellos” (Margaix-Arnal, 2008a).

Aspectos analizados:

\begin{tabular}{|l|}
\hline \multicolumn{1}{|c|}{ Finalidad } \\
\hline -Publica eventos e información \\
-Acceso a recursos de la biblioteca \\
-Se comparten fotos \\
-Se comparten videos \\
\hline \multicolumn{1}{|c|}{ Rasgos } \\
\hline -Incluye enlace a la biblioteca \\
-Antigüedad superior a 1 año \\
-Está en otras redes sociales \\
\hline
\end{tabular}

- Finalidad y rasgos de archivos multimedia compartidos en las bibliotecas universitarias españolas

Los sitios para compartir archivos les permiten a los usuarios que lo deseen subir archivos (audio, vídeo, imágenes, etc.), que pasan a estar disponibles en libre acceso pudiendo ser consultados por multitud de 
usuarios desde cualquier punto geográfico. Los sitios con estas cualidades más empleados por parte de las bibliotecas universitarias españolas son: YouTube, ${ }^{7}$ Slideshare, ${ }^{8}$ IssuuVimeo ${ }^{9}$ o Flickr $^{10}$.

Para Gómez y Merlo (2010) las características principales de estas herramientas son: a) incluir funcionalidades sociales, b) insertar contenidos de otras páginas web, c) configurar los archivos con diferentes niveles de privacidad y licencias de uso.

Aspectos analizados:

\begin{tabular}{|l|}
\hline \multicolumn{1}{|c|}{ Finalidad } \\
\hline -Crear galerías de fotos, presentaciones \\
-Crear canal de videos \\
-Apoyar a los servicios bibliotecarios \\
- llustrar sobre recursos, eventos y actividades \\
-Promoción \\
-Comunicación y participación de usuarios \\
\hline \multicolumn{1}{c|}{ Rasgos } \\
\hline -Incluye más de 4 álbumes, fotos, presentaciones \\
-Incluye más de 4 videos \\
-Empleo de Flickr \\
-Empleo de Picasa \\
-Empleo de Youtube \\
-Empleo de Slideshare \\
-Empleo de otras herramientas \\
\hline
\end{tabular}

\section{- Otros}

Además de los parámetros descritos se ha incluido un espacio para recoger y analizar otros aspectos que aparecen de manera más minoritaria en las web de las bibliotecas universitarias españolas. Los servicios detectados son los que se citan a continuación: servicios de gestión de marcadores (Delicious); escritorios virtuales personalizados (Netvives); webs para publicar historias de manera colectiva (herramienta Meneame); editores visuales (herramienta Pipes), Metaversos $^{12}$ (SecondLife) y microbloging.

7 Sitio web para compartir archivos audiovisuales, http://www.youtube.com

8 Sitio web para compartir presentaciones, http://www.slideshare.com

9 Sitio web para compartir vídeos, http://vimeo.com/issuu

10 Sitio web para compartir fotografías, http://www.flickr.com

11 Servicio para compartir imágenes de Google.

12 Los metaversos son entornos donde los humanos interactúan social y económicamente como iconos a través de un soporte lógico en un ciberespacio que actúa como una metáfora del mundo real, pero sin las limitaciones físicas (Wikipedia). 


\section{Resultados}

\subsection{Recursos de la web 2.0: datos globales}

De las 63 bibliotecas universitarias españolas analizadas, 57, (el $90.4 \%$ ) cuentan con algún recurso de la web 2.0, (Tabla I).

Los recursos más extendidos son:

- Opac 2.0: 43 bibliotecas $(75.4 \%)$

- Redes sociales: 34 bibliotecas $(59.64 \%)$

- Blog: 33 bibliotecas $(57.89 \%)$

- RSS: 29 bibliotecas (50.87\%)

- Compartir archivos multimedia: 13 bibliotecas (22.80\%)

- Mensajería instantánea: 11 bibliotecas (19.29\%)

También se han contabilizado 13 bibliotecas (el 19.69\%) que utilizan otros tipos de recursos, aunque de manera mucho más reducida.

\subsection{La finalidad y los rasgos de los recursos utilizados}

\section{OPAC 2.0}

En la cifra global que se recoge para esta categoría (43 bibliotecas) hay que distinguir entre las 7 bibliotecas universitarias españolas que tienen OPAC 2.0 (en algunas en versión de prueba) —habitualmente manteniendo también el OPAC tradicional-y las 36 bibliotecas que se han limitado a añadir información o elementos del OPAC 2.0 al tradicional. Es muy frecuente que gran parte de los registros no tengan rellena esta información.

Las bibliotecas que cuentan ya con el OPAC 2.0 responden, la mayoría, al producto Encore, de la firma Innovative Interfaces. Su principal cualidad es que permite recuperar la información de forma independiente del Sistema de Gestión Bibliotecaria y aporta mejoras a la búsqueda.

Respecto a los rasgos que destacan (Tabla II) está el empleo de herramientas para buscar en la red (29 bibliotecas); el añadir a los registros la imagen de la cubierta del libro, el sumario, etc. (29 bibliotecas); los enlaces a recursos de información (22 bibliotecas); la creación de listas de libros por los usuarios (21 bibliotecas), y el incluir etiquetas, puntuaciones y comentarios (11 bibliotecas). 
Tabla II. Finalidad y rasgos del OPAC 2.0.

\begin{tabular}{|l|c|c|}
\hline $\begin{array}{c}\text { Finalidad y rasgos del OPAC 2.0 en la bibliotecas } \\
\text { universitarias españolas }\end{array}$ & Núm. de bibliotecas & Porcentaje \\
\hline Finalidad & 36 & 83.72 \\
\hline Añadir información al Opac tradicional & 7 & 16.27 \\
\hline Cambio al Opac 2.0 & 29 & 67.44 \\
\hline Rasgos & 21 & 48.83 \\
\hline Herramientas para buscar en la Red & 11 & 25.58 \\
\hline Usuarios que crean sus propias listas de libros & 29 & 67.44 \\
\hline Incluir etiquetas, puntuaciones y comentarios & 0 & 0 \\
\hline Registros añaden cubierta del libro, sumario, etc. & 22 & 51.16 \\
\hline Ordenación de resultados según su información social & & \\
\hline Enlaces a recursos de información & & \\
\hline
\end{tabular}

\section{Redes sociales}

A excepción de la biblioteca de la Universidad San Pablo CEU, todas las bibliotecas que están presentes en las redes sociales (34 bibliotecas) forman parte de la red Facebook, y 23, además, participan en otras redes (casi siempre Twitter ${ }^{13}$ ). Por ello en este apartado se han analizado la finalidad y los rasgos de la presencia de estas bibliotecas en Facebook. Se trata, además, de la red social de carácter generalista más usada en España, (IAB, 2010).

La finalidad principal es publicar eventos e información ( 33 bibliotecas), también compartir fotos ( 29 bibliotecas) y en menor medida facilitar el acceso a recursos de la biblioteca (18 bibliotecas) — normalmente a la consulta del catálogo — y compartir videos (10 bibliotecas).

Entre los rasgos analizados destaca el hecho de que 24 bibliotecas están presentes con una antigüedad superior a un año. También, habría que destacar que sólo en 7 bibliotecas (de las 34) la presencia en Facebook no es iniciativa de la biblioteca universitaria general sino de determinadas bibliotecas de algunos centros.

Tabla III. Finalidad y rasgos de Facebook.

\begin{tabular}{|l|c|c|}
\hline $\begin{array}{c}\text { Finalidad y rasgos de Facebook en la bibliotecas } \\
\text { universitarias españolas }\end{array}$ & $\mathbf{N}^{\mathbf{0}}$ de bibliotecas & Porcentaje \\
\hline Finalidad & & \\
\hline Publica eventos e información & 33 & 97.05 \\
\hline Acceso a recursos de la biblioteca & 18 & 52.94 \\
\hline Se comparten fotos & 29 & 85.29 \\
\hline Se comparten videos & 10 & 29.41 \\
\hline
\end{tabular}




\begin{tabular}{|l|c|c|}
\hline Rasgos & & \\
\hline Incluye enlace a la biblioteca & 31 & 91.17 \\
\hline Antigüedad superior a 1 año & 24 & 70.58 \\
\hline Está en otras redes sociales & 24 & 70.58 \\
\hline
\end{tabular}

\section{$B \log$}

Los datos de la presente investigación reflejan que el blog es el tercer recurso más difundido (Tabla IV). Se utiliza de manera mayoritaria para informar sobre novedades y noticias de las bibliotecas (27 bibliotecas) y para fomentar la participación y comunicación de los usuarios ( 33 bibliotecas). En la categoría "otros" se contemplan otros fines más concretos y limitados, como es el caso de la Biblioteca de la Universidad de Córdoba y su blog Leyendo Europa que pretende un fin coyuntural:

desde la literatura podamos redescubrir nuestra identidad europea y contribuyamos desde el espacio infinito de la red a subrayar que Córdoba tiene argumentos más que suficientes para convertirse en 2016 en capital europea de la cultura.

También se integra en este apartado la biblioteca de la Universidad de Cantabria con su blog: "Imaginar la ciencia" dedicado al cine, la literatura y la divulgación científica.

La estructura de los blogs recopilados es parecida; por ello presentan unos rasgos coincidentes, entre los cuales destacan la inclusión de la fecha en los post ( 33 bibliotecas), la organización en archivos ( 31 bibliotecas), la posibilidad de recuperar las entradas por fecha ( 31 bibliotecas), por temas y/o categorías ( 31 bibliotecas) y por palabras clave ( 28 bibliotecas), la RSS de las entradas y/o de los comentarios ( 28 bibliotecas).

Entre las bibliotecas que destacan por presentar los blogs más completos destacan: Sevilla, Zaragoza, la Palmas de Gran Canaria, Burgos, Salamanca, Politécnica de Cataluña, Rey Juan Carlos, UNED o Autónoma de Madrid. Y por el número y variedad de blogs: Sevilla, Autónoma de Barcelona, UNED, Politécnica de Madrid o Politécnica de Valencia. 
Tabla IV: Finalidad y rasgos del Blog.

\begin{tabular}{|l|c|c|}
\hline \multicolumn{1}{|c|}{$\begin{array}{c}\text { Finalidad y rasgos del blog en la bibliotecas } \\
\text { universitarias españolas }\end{array}$} & Núm. de bibliotecas & Porcentaje \\
\hline Finalidad & & \\
\hline Apoyo a los servicios bibliotecarios & 28 & 39.39 \\
\hline Informa sobre novedades y noticias de la biblioteca & 33 & 84.84 \\
\hline Participación, comunicación de los usuarios & 0 & 100 \\
\hline Uso interno de los bibliotecarios & 6 & 18.18 \\
\hline Otros & & \\
\hline Rasgos & 19 & 57.57 \\
\hline Tiene más de un blog & 23 & 69.69 \\
\hline Incluye enlace a la página de la biblioteca & 31 & 93.93 \\
\hline Tiene archivos & 28 & 84.84 \\
\hline RSS de las entradas y comentarios & 31 & 93.93 \\
\hline Se pueden buscar las entradas por temas, categorías & 31 & 93.93 \\
\hline Se pueden buscar las entradas por fecha & 28 & 84.84 \\
\hline Las entradas se pueden recuperar por palabras clave & 33 & 100 \\
\hline Hay fecha en los post & 27 & 81.81 \\
\hline Las entradas archivadas superan un año & 9 & 27.27 \\
\hline Los últimos post están entre los dos últimos días & 18 & 54.54 \\
\hline Se incluyen enlaces a recursos relevantes & 15 & 45.45 \\
\hline Incluye enlaces a blogs similares & & \\
\hline
\end{tabular}

\section{RSS}

En el presente análisis se ha detectado que este recurso (Tabla V) se emplea para difundir noticias y eventos de la biblioteca (26 bibliotecas). También, en algunas, para avisar acerca de las novedades del catálogo (8 bibliotecas). Otras bibliotecas disponen de varios canales: la Universidad de Barcelona tienes tres canales de distribución de noticias: uno para novedades, otro para formación y otro para novedades en recursos de información. La Complutense tiene una gran variedad para cada uno de los blog generales, para la actualidad de cada biblioteca y para las novedades del catálogo (uno por cada tipología documental: e-prints, tesis, fondo antiguo, mapas, películas, etcétera).

En cuanto a las características del formato de estos canales, casi todos coinciden en permitir la búsqueda de las noticias (29 bibliotecas), en incluir instrucciones de uso (28 bibliotecas) y en presentar las noticias clasificadas por temas, fecha o título (23 bibliotecas). 
Tabla V: Finalidad y rasgos de RSS.

\begin{tabular}{|l|c|c|}
\hline \multicolumn{1}{|c|}{$\begin{array}{c}\text { Finalidad y rasgos de RSS en la bibliotecas } \\
\text { universitarias españolas }\end{array}$} & Núm. de bibliotecas & Porcentaje \\
\hline Finalidad & 26 & 89.65 \\
\hline Noticias y eventos de la biblioteca & 8 & 27.58 \\
\hline Novedades del catálogo & 2 & 6.89 \\
\hline Otros & & 6.89 \\
\hline Rasgos & 2 & 79.31 \\
\hline Enlaces a páginas para descargar lectores RSS & 23 & 96.55 \\
\hline Noticias clasificadas por temas, fecha o título & 28 & 100 \\
\hline Instrucciones sobre uso & 29 & \\
\hline Las noticias se pueden buscar & & \\
\hline
\end{tabular}

\section{Compartir archivos multimedia}

Como resultado del análisis efectuado (Tabla VI) se comprueba que este recurso se emplea como herramienta de promoción, sobre todo, creando galerías de fotos y/o presentaciones (11 bibliotecas) y también subiendo videos (10 bibliotecas). Su propósito más extendido es ilustrar sobre los recursos, eventos y actividades de la biblioteca (12 bibliotecas) y facilitar la participación de los usuarios (11 bibliotecas). Las aplicaciones más utilizadas son Flickr (9 bibliotecas), Youtube ( 9 bibliotecas) y Slideshare (5 bibliotecas).

Tabla VI. Finalidad y rasgos de compartir archivos multimedia.

\begin{tabular}{|l|c|c|}
\hline $\begin{array}{c}\text { Finalidad y rasgos de compartir archivos multimedia } \\
\text { en la bibliotecas universitarias españolas }\end{array}$ & Núm. de bibliotecas & Porcentaje \\
\hline Finalidad & 11 & 84.61 \\
\hline Crear galerías de fotos, presentaciones & 10 & 76.92 \\
\hline Crear canal de videos & 7 & 53.84 \\
\hline Apoyar a los servicios bibliotecarios & 12 & 92.3 \\
\hline Ilustrar sobre recursos, eventos y actividades & 13 & 100 \\
\hline Promoción & 11 & 84.61 \\
\hline Comunicación y participación de usuarios & & 76.92 \\
\hline Rasgos & 10 & 69.23 \\
\hline Incluye más de 4 álbumes, fotos, presentaciones & 9 & 69.23 \\
\hline Incluye más de 4 videos & 9 & 7.69 \\
\hline Empleo de Flickr & 1 & \\
\hline Empleo de Picasa & 14 & \\
\hline
\end{tabular}

14 Servicio para compartir imágenes de Google. 


\begin{tabular}{|l|c|c|}
\hline Empleo de Youtube & 9 & 69.23 \\
\hline Empleo de Slideshare & 5 & 38.46 \\
\hline Empleo de otras herramientas & 3 & 6.97 \\
\hline
\end{tabular}

\section{Mensajería instantánea}

Cuando este recurso aparece en las bibliotecas analizadas (Tabla VII), está destacado en la web de la biblioteca para que los usuarios le hagan preguntas al bibliotecario, por lo que se puede considerar como ayuda de orientación en general a los servicios de la biblioteca (9 bibliotecas). En la explicación del servicio, en muchas ocasiones se incide en las cuestiones sobre la búsqueda y uso de las fuentes de información, por ello, se ha recogido como otra finalidad más específica el apoyo al servicio de referencia ( 5 bibliotecas, por ejemplo las bibliotecas de las universidades Complutense y San Pablo CEU).

También está el caso de la biblioteca de la UNED que ofrece dos chats: uno general para atención de usuarios con horario de 10 a 19 horas, y otro para información bibliográfica y referencia con horario de 9 a 14 horas.

Se hace notar que la Biblioteca de la Universidad de Cádiz incluye el chat como una herramienta del catálogo para realizar preguntas sobre la búsqueda y acceso a la información a través del mismo chat.

Tabla VII: Finalidad y rasgos de la mensajería instantánea.

\begin{tabular}{|l|c|c|}
\hline $\begin{array}{c}\text { Finalidad y rasgos de la mensajería instantánea en } \\
\text { la bibliotecas universitarias españolas }\end{array}$ & Núm. de bibliotecas & Porcentaje \\
\hline Finalidad & 5 & 45.45 \\
\hline Apoyo al servicio de referencia & 9 & 81.81 \\
\hline Orienta sobre los servicios de la biblioteca & & 100 \\
\hline Rasgos & 11 & 54.54 \\
\hline Chat a base de texto & 6 & 9.09 \\
\hline Chat con voz & 1 & 45.45 \\
\hline Servicio disponible 8 horas al día & 5 & \\
\hline Servicio disponible más de 8 horas al día & & \\
\hline
\end{tabular}

\section{Otros}

Además de los ya referenciados se han localizado otros recursos que están menos representados pero que son propios de la Web 2.0 (Tabla I): servicio de gestión de marcadores (herramienta Delicious), 8 bibliotecas; escritorio virtual personalizado (herramienta Netvibes), 8 bibliotecas; web para publicar historias de forma colectiva (herramienta Meneame), 2 bibliotecas; editor visual (herramienta Pipes), 1 biblioteca; microbloging, 1 biblioteca; Second life, 1 biblioteca. 


\section{Conclusiones}

Después del análisis de los datos se comprueba que aunque el $90.4 \%$ de la bibliotecas universitarias españolas (57 biblioteca de 63) utilizan algún recurso relacionado con la web 2.0, el 17.4 \% (11 bibliotecas de 63) incluye sólo un tipo de recurso y el $25.3 \%$ (16 bibliotecas de 63) sólo dos recursos; por ello se puede afirmar que todavía no existe una gran desarrollo del uso de estos elementos, cuya aplicación por parte de las bibliotecas universitarias puede ser mucho más intensiva.

De entre los recursos de la web 2.0 revisados la transformación del OPAC tradicional en el OPAC 2.0 es la tendencia más extendida y la mensajería instantánea el recurso menos utilizado. El uso del OPAC a través de la web es uno de los grandes logros y principales aportaciones de los servicios bibliotecarios que ofrecen a través de Internet. Las bibliotecas universitarias están atentas a perfeccionar este servicio con los últimos avances, pues se trata de un servicio muy utilizado. No obstante, hay que admitir que un porcentaje elevado de bibliotecas no realizan un planteamiento de este recurso ex profeso para el entorno 2.0, sino que siguen limitándose, en buena medida, a añadir a sus OPAC tradicionales algunas funcionalidades propias de la web 2.0.

Tanto las redes sociales (Facebook en particular) como los blogs son servicios bastante extendidos y de un uso asentado en las bibliotecas universitarias españolas. Aunque el empleo de Twitter comienza a extenderse, Facebook sigue siendo la red usada de manera mayoritaria y un número elevado de bibliotecas vienen participando en esta red desde hace más de un año. El uso principal de Facebook es informativo (publicar eventos e información de interés para los usuarios), así como compartir fotografías. Por su parte los blogs se ven, en mayor grado, como elemento para la participación y comunicación de los usuarios. Esta diferenciación resulta llamativa y puede deberse a que, aun siendo las redes sociales vehículos ideales para la interacción con los públicos, el origen de las bitácoras es anterior (Barger, 1999) y, por tanto, son soportes que están más consolidados. No obstante, dadas las características de Facebook y su utilidad como herramienta para interconectar públicos, se prevé un incremento del empleo de las redes sociales por parte de las bibliotecas en este sentido.

Las fuentes RSS se utilizan sobre todo para difundir noticias y eventos de la biblioteca. Y de forma mucho más limitada, para dar a conocer las novedades del catálogo bibliotecario. Se aprecia aquí una potencialidad que se está dejando de aprovechar por parte de las bibliotecas universitarias españolas, que podrían valerse en mayor medida de estas fuentes para hacerles llegar a sus usuarios las obras recién incorporadas a sus catálogos. 
La creación y gestión de los archivos multimedia suele requerir de una mayor dedicación temporal y un presupuesto superior al de la puesta en marcha de otros recursos 2.0 (como los blogs, por ejemplo). Por esta razón se interpreta que su uso, especialmente el de los canales de video, es más reducido. La principal finalidad de este tipo de archivos suele ser promocional. Se trata, tanto en el caso de los videos como en el de las fotografías y presentaciones, de un recurso con un valor más comercial que informativo y puede ser de especial relevancia para la imagen y la reputación de las bibliotecas universitarias.

Por su parte la mensajería instantánea, el menos común de los factores analizados, se emplea, en gran medida, como elemento de orientación sobre los servicios de las bibliotecas.

En términos generales se aprecia un contraste entre un reducido grupo de bibliotecas, el $9.5 \%$ (6 bibliotecas de las 63) que cuenta con una presencia destacada de las categorías de recursos web 2.0 que se han utilizado frente a la mayoría con una presencia más baja, que se limita a integrar algún recurso en la web, quizá porque es la moda, porque las bibliotecas importantes lo hacen. Estas 6 bibliotecas corresponden a destacadas universidades (Sevilla, A Coruña, Complutense, Barcelona, Rey Juan Carlos, Politécnica de Valencia). Estos datos indican que a pesar de existir una preocupación por la incorporación de las herramientas de la web 2.0, el uso que las bibliotecas universitarias españolas hacen de las mismas es bastante incipiente.

En función de los resultados analizados se aprecia que el planteamiento que las instituciones analizadas hacen de los recursos 2.0 es de carácter más informativo que comunicativo. Únicamente los blogs, quizás por su mayor asentamiento temporal, se plantean más como recursos de comunicación y participación de los públicos. Por este motivo, y dado el enorme potencial que tienen las herramientas de la web 2.0 para agilizar las conversaciones entre las organizaciones y sus públicos, se considera que se están dejando de aprovechar las posibilidades de interacción que brindan estos soportes comunicativos. Todo este potencial comunicativo, convenientemente gestionado, proporcionaría las claves para una adecuada imagen de reputación enlínea de las instituciones.

Es posible afirmar que los recursos de la web 2.0 seguirán incrementándose en un futuro inmediato en esta bibliotecas, pues entre el corto periodo comprendido entre los dos momentos en los que se analizó el estado de los sitios web, ha habido nuevas incorporaciones. Por ejemplo, la biblioteca de la Universidad de Zaragoza pasó de no tener ningún recurso a presentar de forma destacada varias herramientas 2.0 como una novedad. En este sentido los datos de esta investigación servirán en un futuro para contrastarlos y 
compararlos con los de otra que vuelva a analizar cómo se ha incrementado y evolucionado la presencia de estos recursos.

Por todo lo expuesto anteriormente, y volviendo a las cuestiones que fundamentaban los objetivos de esta investigación, habría que concluir que el uso que las bibliotecas universitarias españolas hacen de los recursos 2.0 es bastante incipiente. Hay unas pocas universidades que los emplean con cierta profusión, mientras que la gran mayoría hace un uso bastante limitado de ellos y mantiene un planteamiento más informativo que relacional. Por tanto, es recomendable que estas organizaciones exploten el potencial de interacción con los públicos que pueden proporcionar estas herramientas y, de manera subsidiaria, las empleen para gestionar y promocionar su imagen y la reputación en el contexto en-línea.

Como posibles líneas futuras de investigación, el presente trabajo, basado en el análisis de contenido, se podría completar con otro estudio que tenga como objetivo comprender la influencia de los efectos de estas herramientas en las bibliotecas universitarias, por ejemplo, mediante preguntas a través de un panel Delphi realizado por especialistas o a través de estudios de casos.

\section{REFERENCIAS BIBLIOGRÁFICAS}

Abram, Stephen (2005), Web 2.0, library 2.0 and librarian 2.0: preparing for the 2.0 world [en línea], [consulta: 6 agosto 2011], disponible en: http://www.imakenews.com/sirsi/e_article00050568 8.cfm

Abram, Stephen (2006), Podcasts and libraries [en línea], [consulta: 6 agosto 2011], disponible en: http://stephenslighthouse.sirsidynix. com/archives/2006/11/podcasts_and_li.html

Andreu Felipe, Isabel; Brugarolas Ros, Carmen María; Alcázar Ruiz, Concepción D.; Cárceles Andreu, José Luis (2007), “Aplicaciones de los blogs en unidades de información: usos y perspectivas”, en Scire, vol. 13, núm 1, enero-junio, pp. 133-144.

Barger, Jom (1999), FAQ: Weblog resources [en línea], [consulta: 9 agosto 2011], disponible en: http://www.robotwisdom.com/weblogs/index.html

Boeninger, Chad (2006), "Using a wiki as a research guide: a year's experience”, en Library Voice, july, [consulta: 3 septiembre 2011], disponible en: http://libraryvoice.com/wikis/using-a-wiki-as-aresearch-guide-a-years-experience

Borrell, Marina; Maciel, Evangelina (2009), "Implementación de un servicio de referencia virtual por chat en una biblioteca universitaria argentina", en Revista Española de Documentación Cientifica, vol. 32, núm 4, octubre-diciembre, pp. 133-143. 
Boschma, Jeroen (2006), Generación Einstein: inteligentes, sociales y superfast, Communicating with Young People in the 21st Century, Pearson Education Benelux, Schiedam.

Breeding, Marshall (2007), "We need to go beyond Web 2.0", en Computers in Libraries, vol. 27, núm 5, pp. 22-25.

Cant, A. G. (2004), "Internationalizing the business curriculum: developingintercultural competence", en Journal of American Academy of Business, vol. 5, núm 1/2, pp.177-182.

Casey, Michael E.; Savastinuk, Laura C. (2006), “Library 2.0”, en Library journal, vol. 131, núm 14, septiembre, pp. 40-42, también disponible en: http://www.libraryjournal.com/article/CA636520 0.html

Castillo Díaz, Ana (2007), La comunicación de las marcas universitarias españolas a través de sus sedes webs institucionales, tesis doctoral. Universidad de Extremadura.

Celaya, Javier (2008), La empresa en la web 2.0. El impacto de las redes sociales y las nuevas formas de comunicación online en la estrategia empresarial, Barcelona: Gestión 2000.

Celaya, Javier; Herrera, Pablo (2007), Los blogs en la comunicación empresarial en España, Madrid: BMPO.

Chad, Ken; Miller, Paul (2005), Do libraries matter?: The rise of library 2.0 [en línea], [consulta: 10 septiembre 2011], disponible en: http://www.talis.com/applications/downloads/white_papers/ DoLibrariesMatter.pdf.

Chen Xu, Fenfei Ouyang and Heting Chu, "The Academic Library Meets Web 2.0: Applications and Implications", en The Journal of Academic Librarianship, vol. 35, núm 4, pp. 324-331.

Chua, Alton Y. K; Goh, Dion H. (2010), "A study of Web 2.0 applications in library websites", en Library E Information Science Research, vol. 32, pp. 203-211.

Coombs, Karen A. (2007), "Building a library web site on the pillars of Web 2.0", en Computers in Libraries, vol. 27, núm 1 [consulta: 6 agosto 2011], disponible en: http://www.infotoday.com/cilmag/ jan07/Coombs.shtml

Dobrecky, Leticia Paula (2007), "Hacia la library 2.0: blogs, rss y wikis”, en El profesional de la información, vol. 16, núm 2, marzoabril, pp. 138-142.

Fichter, Darlene (2006), "Using wikis to support online collaboration in libraries", en Information Outlook, vol. 10, núm 1, pp. 30-1.

Foley, Marianne (2002), "Instant messaging reference in an academic library: a case study", en College and Research Libraries, vol. 63, pp. 36-45.

Geoghegan, Michael W.; Klass, Dan (2005), Podcast Solutions: The Complete Guide to Podcasting, Berkeley, CA: Friends of ED.

Gómez Pereda, Noemí; Merlo Vega, José Anatonio (coords.) (2010), "Dossier: Experiencias bibliotecarias con las tecnologías sociales", en Educación y Biblioteca, vol. 177, mayo-junio, pp. 61-125. 
González Fernández-Villavicencio, Nieves (2007), “Bibliotecas 2.0 en España (el camino recorrido), en Boletín de la Asociación Andaluza de Bibliotecarios, núm. 86-87, enero-junio, pp. 29-46.

Habib, Michael C. (2006), Toward Academic Library 2.0: Development and Application of a Library 2.0 Methodology. A Master's Paper for the M.S. in L.S degree [en línea], [consulta: 4 agosto 2011], disponible en: http://etd.ils.unc.edu/dspace/handle/1901/356.

Han, Zhiping; Quan Liu, Yan (2010), "Web 2.0 applications in top Chinese university libraries”, en Library Hi Tech, vol. 28, núm 1, pp. 41-62.

Harinarayana, N.S.; Vasantha Raju, N. (2010), "Web 2.0 features in university library web sites", en The Electronic Library, vol. 28, núm 1, pp.69-88.

Harinarayana, N.S.; Kumbar, Mallinath; Chairman, Pradeep P. (2007), "RSS-based information services in libraries: a study", en Library Herald, vol. 45, núm. 2, pp. 130-9.

IAB (2010), II Estudio sobre redes sociales en Internet [en línea], [consulta: 6 septiembre 2011], disponible: http://www.iabspain.net/ ver.php? $\bmod =$ noticias $\&$ identificador $=80$

Jowitt, Angela (2007), "Perceptions and usage of library instructional podcasts by staff and students at Universal College of Learning (UCOL)", en Support Services Dissertations and Theses, Paper 1 [en línea], [consulta: 4 agosto 2011], disponible en:http://www.coda. ac.nz/ucol_ss_di/1

Leiva Aguilera, Javier (2006), "Blogs: una herramienta de difusión para profesionales de la información”, en TK, núm. 18, diciembre, también disponible en: http://www.documentalistaenredado. net/contenido/blogstk.pdf

Linh, Nguyen Cuong (2008), "A survey of the application of Web 2.0 in Australasian university libraries", en Library Hi Tech, vol. 26, núm 4, pp. 630-53.

Liu, Shu (2008), "Engaging users: the future of academic library web sites”, en College ER Research Libraries, vol. 69, núm 1, pp. 6-27.

Maness, Jack M. (2006), "Library 2.0 theory: Web 2.0 and its implications for libraries", en Webology, vol. 3, núm 2 [en línea], [consulta: 4 octubre 2011], disponible en: http://www.webology.ir/2006/ v3n2/a25.html

Margaix-Arnal, Didac (2007), “Conceptos de web 2.0 y biblioteca 2.0: origen, definiciones y retos para las bibliotecas actuales", en Elprofesional de la información, vol. 16, núm 2, marzo-abril, pp. 95-106.

Margaix-Arnal, Didac (2008a), "Las bibliotecas universitarias y Facebook: cómo y por qué estar presentes”, en El profesional de la información, vol. 17, núm. 6, noviembre-diciembre, pp. 589-601.

Margaix-Arnal, Didac (2008b), El OPAC 2.0: Puerta de Acceso a los Contenidos de la Biblioteca, 2008, en IV Congreso Nacional de Bibliotecas Públicas, A Coruña (España), 24-26 de septiembre de 2008, Madrid: Ministerio de Cultura, pp. 237-246. 
Marín Ruiz, Antonio, et al. (coords.) (2005), Mass media y Universidad. El reto de la comunicación en las universidades, Granada: SOMEDICYT, Universidad de Granada.

Merlo Vega, José Antonio (2005), "Servicios públicos de referencia en línea", en BID. Textos universitaris de bibliotecnomoia i documentació, núm. 14, [consulta: 4 agosto 2011], disponible en: http:// www2.ub.es/bid/consulta_articulos.php?fichero $=14 \mathrm{merlo} 2 . \mathrm{htm}$

O'Reilly, Tim (2005), What is Web 2.0: Design patterns and business models for the next generation of software? [en línea], [consulta 24 julio 2009], disponible en: http://oreilly.com/web2/archive/whatis-web-20.html

Ríos Castillo, Luis Eduardo (2009), Biblioteca 2.0 [en línea], [consulta: 4 agosto 2011], disponible en: http://repositorio.oui-iohe.org/ dspace/bitstream/123456789/1530/1/Biblioteca_2_0_newVersion.pdf 